\title{
Distribución espacial y temporal de aprovechamientos de agua del acuífero del Valle de Tecamachalco, Puebla
}

Spatial and Temporal Distribution of the Water Exploitation in the Aquifer Valle de Tecamachalco, Puebla

Lucia Hernández-Vivanco

Colegio de Postgraduados, México

ORCID: http://orcid.org/0000-0003-0959-0732

Luis Alberto Villarreal-Manzo a

Colegio de Postgraduados, México

lvillarrealm@hotmail.com

ORCID: http://orcid.org/0000-0002-3085-2082

Benito Ramirez-Valverde

Colegio de Postgraduados, México

ORCID: http://orcid.org/0000-0003-2482-5667

Ignacio Ocampo-Fletes

Colegio de Postgraduados, México

ORCID: http://orcid.org//0000-0001-6311-1072

José Luis Jaramillo-Villanueva

Colegio de Postgraduados, México

ORCID: http://orcid.org/0000-0001-8179-6351

Benjamin Ortiz-Espejel

Universidad Iberoamericana Golfo Centro-Puebla, México

ORCID: http://orcid.org/0000 000162016452
DOI: https://doi.org/10.11144/Javeriana.ayd22-42.deta Redalyc: http://www.redalyc.org/articulo.oa? id $=151557418002$

Fecha de recepción: 20 Agosto2017 Fecha de publicación: 20 Julio 2018

\section{Resumen:}

La presente investigación se realizó con el propósito de analizar la distribución geoespacial de los aprovechamientos de agua del Acuífero del Valle de Tecamachalco Puebla, México, a fin de identificar zonas de mayor densidad de aprovechamientos hídricos e intensidad del uso del agua. A partir de datos del Registro Público de Derechos de Agua de la Comisión Nacional del Agua, de 1994 a 2014 se obtuvieron 1286 aprovechamientos, con un volumen concesionado de 194223870.9 metros cúbicos anuales. El análisis espacial mediante el cálculo de la densidad tipo Kernel permitió determinar la probabilidad de ocurrencia y localización en el espacio de los aprovechamientos hídricos o pozos profundos, además de su diferenciación geográfica en el acuífero en función de su distribución. Con este método se generaron mapas de tipo isoplético, que representan conjuntos de puntos en el espacio. Conforme los resultados, se encontró que predominan, en un 78\%, los pozos de uso agrícola; seguido por los de uso público-urbano, con un 16,7\%. El patrón de distribución muestra que las áreas de mayor densidad de pozos profundos se ubican en los municipios de Palmar de Bravo y Quecholac, e indica las zonas de mayor extracción.

Palabras clave: agua subterránea, pozos, densidad Kernel, uso del agua.

\begin{abstract}
:
This research was conducted in order to analyze the geospatial distribution of the water exploitation in the aquifer Valle de Tecamachalco, Puebla, México, aiming to identify the zones with higher water exploitation density and higher water use intensity. Based on data from the Water Right Public Records of the Water National Commission, taken from 1994 to 2014, 1 286 exploitation points were identified with a licensed volume of 194223870.9 cubic meters per year. Spatial analysis through the kernel density estimation allowed to determine the occurrence probability and spatial location of either water exploitations points or deep wells. It also allowed the geographic differentiation within the aquifer based on their distribution. This method enabled to produce isopleth maps that represent sets of points in the space. The outcomes indicated a $78 \%$ prevalence of wells for farming use, followed by those for public-urban use with $16.7 \%$. The distribution pattern shows that the areas with higher density of deep wells are located in the towns Palmar de Bravo and Quecholac and also indicates the higher extraction zone. Keywords: underground water, wells, kernel density, water use.
\end{abstract}




\section{Introducción}

Actualmente, tanto la actividad económica como el desarrollo social en muchas regiones del mundo, se ven limitadas por la disponibilidad de agua. Dado que esta se utiliza de manera directa o indirecta en cualquier proceso o actividad humana, se hace evidente una mayor competencia entre los usuarios y una baja disponibilidad de este vital recurso, problemática cuyo origen no solo se debe a su escasez, sino también a su mal manejo. Dicha situación se convierte en un desafío mundial (Guzmán-Soria et al., 2009; Llamas, Aldaya, Garrido, \& López-Gunn, 2009; Barrionuevo \& Feler, 2014).

La sobreexplotación del vital líquido en un territorio, ante su creciente demanda, ha disminuido la disponibilidad del recurso más allá de la capacidad de recarga de los sistemas hídricos, lo que ha afectado a especies animales y vegetales ante las transformaciones de los ecosistemas, y ha causado daños irreversibles en los mismos (Instituto Nacional de Ecología [INE], 2005). En consecuencia, se genera una lucha por el recurso, que presenta un estrés hídrico; es decir, su disponibilidad es cada vez menor y más vulnerable, lo que pone en riesgo la vida en el planeta.

En México, la disponibilidad media de agua por habitante ha ido disminuyendo. En 1950, se contaba con $18035 \mathrm{~m}^{3}$; para el año 2000, fue de $4900 \mathrm{~m}^{3}$; en el 2004, de $4547 \mathrm{~m}^{3}$; en el 2010, de $4090 \mathrm{~m}^{3}$; en el 2013, de $3982 \mathrm{~m}^{3}$; y se estima que para el 2025, descienda hasta $3822 \mathrm{~m}^{3} /$ hab/año (Carabias, Collado, Martínez, \& Landa, 2005; Comisión Nacional del Agua [Conagua], 2014b). La recarga media de los acuíferos es de 77 $\mathrm{km}^{3}$, y se estima un aprovechamiento de $27,4 \mathrm{~km}^{3} /$ año. Es importante destacar que alrededor del $35 \%$ de agua que se extrae es de origen subterránea y, aproximadamente, el $55 \%$ se extrae de acuíferos sobreexplotados. Con esta agua se atiende a más de dos tercios de la población y se abastece, en una tercera parte, a la superficie total irrigada. De igual manera, es utilizada para diversas actividades: $77 \%$ para usos agropecuarios, $13 \%$ para abastecimiento público y 10\% para la actividad industrial (Conagua, 2014a).

Es evidente que la disponibilidad de agua subterránea es un tema crítico en el mundo, además de otros factores que influyen en su agotamiento como la contaminación, los cambios de uso de suelo y la pérdida de zonas de recarga. De acuerdo con los resultados de estudios recientes, se define si los acuíferos se convierten en sobreexplotados o si dejan de serlo, en función de la relación extracción/recarga; es decir, el cambio en el volumen de los caudales y su dinámica de abastecimiento (INE, 2005; Conagua, 2014b).

En este sentido, México presenta una crisis de sobreexplotación de sus acuíferos. En 1975, existían un total de 653 acuíferos, de los cuales 32 eran sobreexplotados; 80 en 1985, 101 en el 2008 y, actualmente, son 106 (Conagua, 2014a). La reserva de agua subterránea del país se está minando a un ritmo de casi $6 \mathrm{~km}^{3}$ por año. De tal manera que la sobreexplotación de los acuíferos es uno de los problemas más importantes para el manejo de agua subterránea (Gutiérrez-Carrillo, Palacios-Vélez, Peña-Díaz, \& Palacios-Vélez, O., 2002). Además, hay ineficiencia en el uso del recurso, principalmente en el sector agropecuario, en el cual se pierde cerca del 60\%, particularmente durante la conducción (Carabias et al., 2005).

El Acuífero del Valle de Tecamachalco (AVT) pertenece a la Región Hidrológica IV Balsas, una de las más importantes de México ya que alberga a los estados con mayor población del centro del país. En 2002, el acuífero presentaba un déficit de agua de -32091151 millones de metros cúbicos anuales (-32091151 $\mathrm{hm}^{3}$ ); para el 2013, este incrementó a -46779564 millones de metros cúbicos anuales $\left(-46779564 \mathrm{hm}^{3}\right)$; y, actualmente, es de -49102251 millones de metros cúbicos anuales $\left(-49102251 \mathrm{hm}^{3}\right)$, mientras que la recarga media anual es de 157,1 millones de $\mathrm{m}^{3}\left(157,1 \mathrm{hm}^{3}\right.$ ). (Conagua, 2003, 2009; Secretaría de Medio Ambiente y Recursos Naturales [Semarnat], 2011, 2015). Actualmente se ha decretado, casi en su totalidad, en veda y no hay autorización para la perforación de nuevos pozos. Sin embargo, el problema persiste, aunado a la contaminación ocasionada por descargas de aguas residuales de algunas industrias instaladas en 
la zona, demás de las aguas residuales domiciliarias, que se vierten a barrancas y generan contaminación de los mantos freáticos.

Para cumplir con el objetivo de analizar la distribución geoespacial de los aprovechamientos de agua subterránea del Acuífero del Valle de Tecamachalco, Puebla, México, se identificaron zonas de mayor concentración de aprovechamientos hídricos, y, con ello, zonas de mayor intensidad del uso del agua subterránea. Se definieron patrones geoespaciales a partir de la determinación de la densidad Kernel de los pozos profundos ubicados en la zona de estudio, para evidenciar la diferenciación geográfica de los aprovechamientos en el AVT y la representación espacial de las variaciones de la densidad de los mismos. El método de Kernel se aplicó con sistemas de información geográfica (SIG), dado que son de gran utilidad para el análisis del territorio por medio de la observación espacial, que permite identificar componentes del territorio y su funcionalidad para representar el comportamiento de un determinado fenómeno. En el estudio se enfatizó en la importancia del agua como uno de los recursos naturales más importantes para la vida, el cual es fundamental para el desarrollo de una sociedad, aun cuando sus fuentes de abastecimiento, como los acuíferos, se encuentran seriamente deterioradas (Conagua, 2014a).

\section{Metodología}

\section{Zona de estudio}

Conagua agrupó a los acuíferos del país en 13 regiones hidrológicas administrativas. El estado de Puebla pertenece a la Región Hidrológica IV Balsas, conformada por 42 acuíferos, de los cuales dos están sobreexplotados. Tal es el caso del Acuífero del Valle de Tecamachalco, localizado en la porción central del estado de Puebla, a unos 20 kilómetros al oriente de la ciudad de Puebla, con una extensión superficial aproximada de $3600 \mathrm{~km}^{2}$. Geográficamente, está limitado por los paralelos $18^{\circ} 32^{\prime \prime} 20.1^{\prime \prime}$ y 19 13’ 45.8” de latitud Norte, y por los meridianos $97^{\circ} 14^{\prime} 31.7^{\prime \prime}$ y $98^{\circ} 3$ ' 6.2” de longitud Oeste (figura 1). Es identificado con la clave 2101 (Conagua, 2009). Política y económicamente, lo conforman 27 municipios con una población total de 728878 habitantes, hasta el año 2010 (Instituto Nacional de Estadística y Geografía [Inegi], 2010).

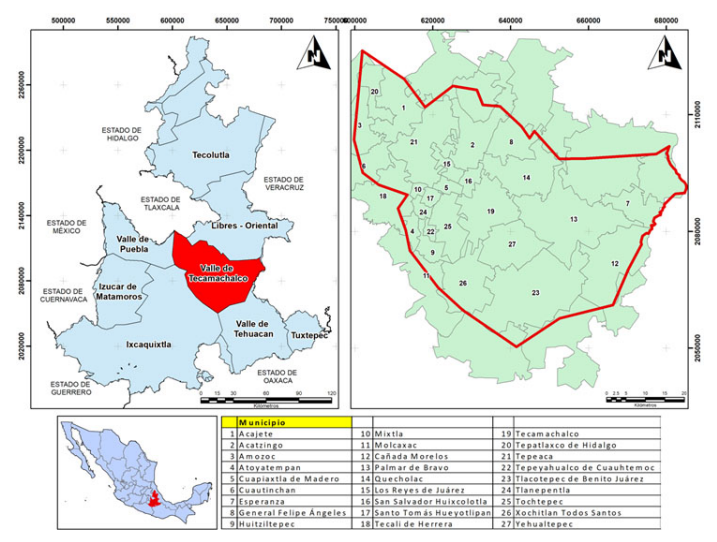

FIGURA 1.

Ubicación de la zona de estudio: Acuífero del Valle de Tecamalco y los acuíferos del estado de Puebla. Fuente: elaboración propia

En la región se identifican varios tipos climáticos: la parte oriental está representada por un clima de tipo semiseco; en la porción central, del tipo templado subhúmedo; y hacia la parte noroccidental, los climas dominantes corresponden a tipos templados subhúmedos. La temperatura media anual es de $16,5^{\circ} \mathrm{C}$. Las zonas más frías se localizan en las faldas de los volcanes, donde la temperatura media fluctúa entre los 12 y $14{ }^{\circ} \mathrm{C}$ (Conagua, 2009). La evaporación potencial promedio es de $1450 \mathrm{~mm} /$ año, la precipitación media es 
de 602 milímetros ( $\mathrm{mm}$ ) y varía de $353 \mathrm{~mm}$ en las zonas de valle, a $850 \mathrm{~mm}$ en las zonas cercanas al Pico de Orizaba y el volcán La Malinche. En la región existe un déficit promedio de humedad de aproximadamente $848 \mathrm{~mm}$. Las primeras lluvias se registran en el mes de mayo y la temporada generalmente termina a principios de octubre.

El valle carece de corrientes superficiales importantes. Solamente se destaca la barranca conocida como "El Águila”, tributaria del río Atoyac, lo cual hace más vulnerable a esta zona ante un estrés hídrico (Conagua, 2003). Por una parte, en la actualidad la región presenta un déficit importante en la disponibilidad de recursos hídricos subterráneos, situación que pone en peligro el abastecimiento de agua para los 499402 habitantes de los 27 municipios que conforman el acuífero (Inegi, 2010). Por otra, se estima un crecimiento de la población para el año 2030 de 900000 habitantes (180\% más de la actual población). Esta situación incrementará la demanda de agua y, en consecuencia, se estima que habrá un desequilibrio entre la extracción y la recarga del orden de $120 \mathrm{hm}^{3} /$ año, lo que se traduce en abatimientos importantes en la zona, sobre todo en las partes del acuífero donde se concentra la extracción, como las zonas cercanas a Tepeaca y Palmar de Bravo (Conagua, 2009).

Otro de los problemas del acuífero es la contaminación causada por la falta de saneamiento de las escasas corrientes superficiales como las barrancas y preponderantemente el río Atoyac, y a la ausencia de plantas de tratamiento en estas corrientes y en aquellos cauces que conducen aguas residuales. Estudios de la Conagua revelan que las barrancas y los terrenos son usados para descargar desechos de Tecamachalco, Acajete, Acatzingo y Tepeaca; estos contaminantes se filtran al subsuelo y provocan contaminación del agua subterránea. A su vez, también están identificadas las industrias como los principales responsables de este problema, pues ocupan al menos 278 sitios para descargar aguas residuales (Conagua, 2009).

\section{Métodos y técnicas de investigación}

La definición de patrones geoespaciales y la densidad de los aprovechamientos de agua subterránea del AVT se obtuvieron a partir de métodos y procedimientos aplicados, los cuales se describen a continuación.

Para ubicar las áreas de mayor presión por extracción de agua en función de la concentración de pozos y volúmenes concesionados, se utilizó información del Registro Público de Derechos de Agua (Repda) y del Localizador del Repda (Locrepda) de la Conagua. Los datos, que van al 31 de diciembre de 2014, contienen la ubicación del pozo, municipio, uso del agua, volumen concesionado, representante y títulos de concesión.

Se utilizó un SIG como herramienta para procesar y analizar los datos. Un primer paso consistió en la conversión de los datos de formato KMZ a formato SHP, para determinar el grado de distribución o concentración de los pozos en el espacio y su relación con otros elementos. El análisis geoespacial mediante el cálculo de la densidad tipo Kernel ha sido ampliamente utilizado, principalmente en investigaciones médicas y sociales, y ha determinado, por ejemplo, focos de incidencia de enfermedades, análisis de problemas sociales y urbanos, entre otros.

La estimación de la densidad Kernel está definida como un procedimiento no paramétrico utilizado para obtener la función de probabilidades de una o más variables. Consiste en determinar la probabilidad de ocurrencia de un determinado evento y su localización en el espacio, y es aplicado a una extensa cantidad de datos y variables en análisis de gran escala regional o global. Los puntos que representan los datos tienen un valor de densidad considerando la distancia que hay entre cada uno de ellos, es decir, a partir de un radio determinado por las características del hecho o fenómeno que se desea analizar (Gibin, Longley, \& Atkison, 2007). El procedimiento considera dos elementos básicos: poblaciones y la superficie de referencia (hectáreas o kilómetros), es decir, una serie de datos localizados en coordenadas longitud y latitud X y Y.

Mediante la visualización de los resultados, se genera una cuadrícula sobre el mapa de isopletas o isodensidades (líneas de contorno), que atribuye, para cada celda, un color específico que corresponde a 
la probabilidad de existencia de cierta concentración de puntos (Moreno, 1991). En general, este color corresponde a un valor y representa una escala continua que va desde colores oscuros a otros más claros, que indican mayor o menor concentración, respectivamente. De manera general, la densidad Kernel es la frecuencia diferencial de un hecho geográfico que se produce en un espacio y tiempo determinados.

En este trabajo se utilizaron los valores predeterminados por el sistema para una mejor visualización de la densidad, a partir de los siguientes parámetros de control del SIG, los cuales fueron utilizados, también, por Gatrell, Bailey, Diggle y Rowlingsont (1996).

Radio de Búsqueda: determina la distancia dentro de la cual se totalizarán los valores y la superficie para obtener la densidad; cuanto mayor sea el radio, se obtendrán patrones más generales. Si se utiliza un radio más pequeño, puede que no se aprecien las variaciones espaciales de la densidad.

Tamaño del Pixel: determina el grado de la finura con el que se representarán las áreas. A mayor nivel de resolución, mayor tiempo de proceso y espacio de almacenamiento. En cambio, si los pixeles son demasiado grandes, los patrones o marcación de áreas pueden llegar a desaparecer. Se recomienda un tamaño entre 10 y 100 pixeles por unidad de densidad.

Unidades: permite establecer la unidad por la que se desea calcular la densidad km, ha, etc.

\section{Resultados}

La presente investigación se centró en el registro de datos de aprovechamientos de agua subterránea (pozos profundos), de 1994 a 2014, del Registro Público de Derechos de Agua y su localizador (ambos de la Conagua), que contienen información de los pozos ubicados en todo el país. En la figura 2 se puede observar la ubicación de los pozos profundos del estado de Puebla, aunque geográficamente algunos de ellos no correspondan a este, ya que sus coordenadas los ubican en otros estados de la república e inclusive en el Golfo de México y en el Océano Pacífico. Esta situación también se presenta en el resto de la información del país, al existir una discrepancia entre los datos y las bases de datos de la misma Conagua.

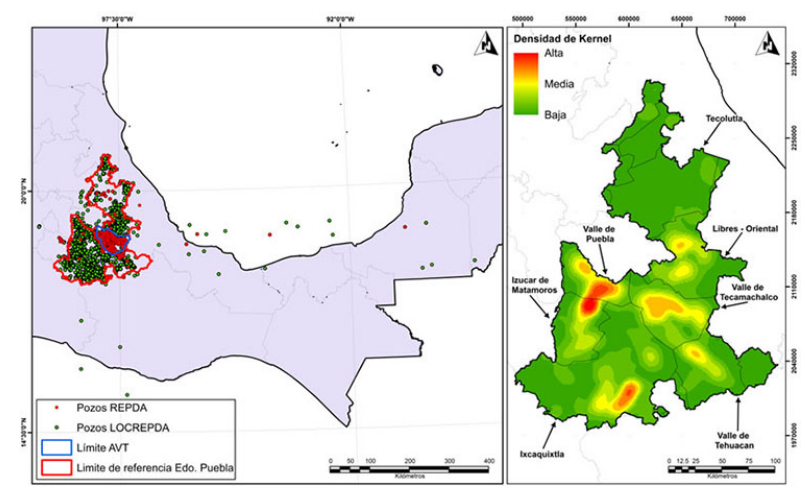

FIGURA 2.

Localización de aprovechamientos de agua subterránea con datos del Repda y Locrepda en el estado de Puebla, y estimación de densidad Kernel para el estado de Puebla.

Fuente: elaboración propia con información del Repda de la Conagua

Derivados de la estimación de densidad Kernel, los aprovechamientos de agua subterránea en todo el estado de Puebla muestran el patrón de distribución geográfico de mayor densidad hacia el centro del estado, que corresponde al área de influencia del Acuífero del Valle de Puebla, el cual concentra a los municipios de mayor población (municipio de Puebla con 1539819, San Martín Texmelucan con 141112 habitantes, San Pedro Cholula con 120459 habitantes, y San Andrés Cholula con 100439 habitantes). Lo mismo ocurre con el Acuífero de Izúcar de Matamoros, cuyos principales centros de población son Atlixco, con 127062 habitantes; e Izúcar de Matamoros, con 72799. (Inegi, 2010), que en total representan el 36\% de la población total del estado de Puebla. Aun cuando existen otras áreas, como la mixteca, donde se hace 
notable el incremento del uso del agua subterránea, se evidencia una alta presión sobre el agua subterránea de los acuíferos en el estado.

Particularmente para esta investigación, se seleccionaron los pozos ubicados dentro del polígono del AVT, conformado por 27 municipios, lo que requirió una depuración de pozos duplicados que se totalizaron en una sola base de datos, con un total de 1286 aprovechamientos. Los resultados del estudio se presentan en cinco periodos, tomando como punto de partida el año 1994, en el que, derivado de la Ley General de Aguas Nacionales, surge el Repda con el fin de inscribir títulos de concesión y asignación de aguas nacionales. Se continuó con el análisis durante los periodos de 1995 a 1999, de 2000 a 2004, de 2005 a 2009 y de 2010 a 2014; lo que mostró la continuidad geográfica, es decir, la dinámica espacial y temporal de los aprovechamientos.

La estimación de la densidad Kernel identificó el patrón de distribución y agrupación de aprovechamientos de agua considerando solo la ubicación geográfica de los pozos (longitud y latitud) y su vecindad con otros pozos, como se mencionó con anterioridad. De acuerdo a los periodos indicados, se observa el comportamiento y evolución espacio - temporal de los aprovechamientos de agua. Para el periodo 1995 a 1999, el registro de pozos se incrementa y la mayor densidad (áreas de mayor concentración de pozos) se observa en los municipios de Quecholac y Palmar de Bravo. De igual manera, se evidencia una mayor dispersión de los pozos.

En los periodos siguientes se observa un incremento en las áreas de mayor densidad que se distribuyen hacia los municipios de Acatzingo, Los Reyes de Juárez y Palmar de Bravo. Se destaca la relación de estas áreas de mayor densidad con el volumen concesionado por la Conagua para la explotación del vital líquido, el cual oscila entre el 30 y $40 \%$ respecto del total concesionado para esos periodos en la región de estudio (figura 3).

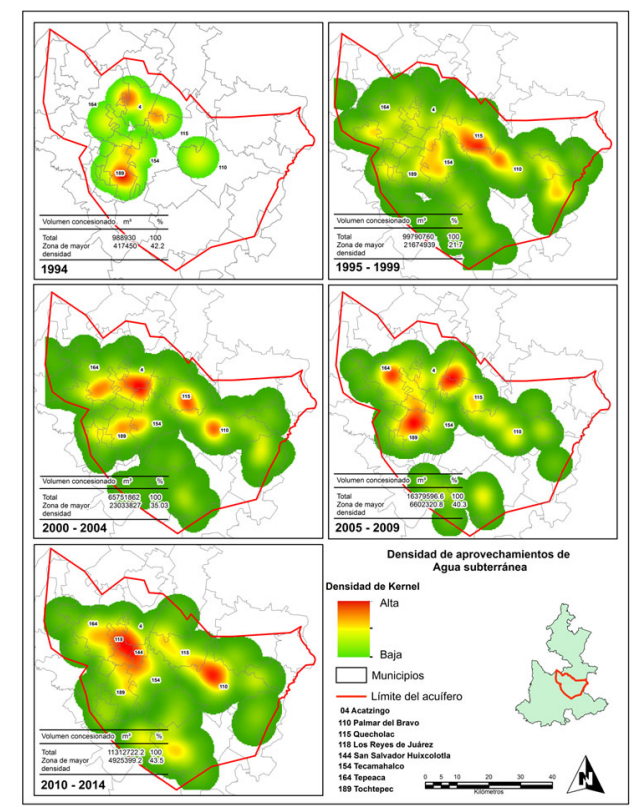

FIGURA 3.

Patrones de distribución espacial de aprovechamientos de agua subterránea del AVT en cinco momentos del periodo analizado.

Fuente: elaboración propia con información del REPDA de la Conagua.

Finalmente, en respuesta al análisis geoespacial, y para destacar las áreas de mayor densidad total de 1994 a 2014, en el cual el límite administrativo municipal no es restrictivo o determinante para un mayor aprovechamiento, en la figura 4 se realiza un acercamiento que refleja que los municipios de Palmar de Bravo y Quecholac corresponden a la región que concentra el mayor número de pozos, es decir, el mayor número de aprovechamientos de agua subterránea. Esta área refleja una mayor presión sobre el vital líquido como 
consecuencia de las actividades productivas, esencialmente agrícolas, que se realizan en los municipios de la zona de estudio.

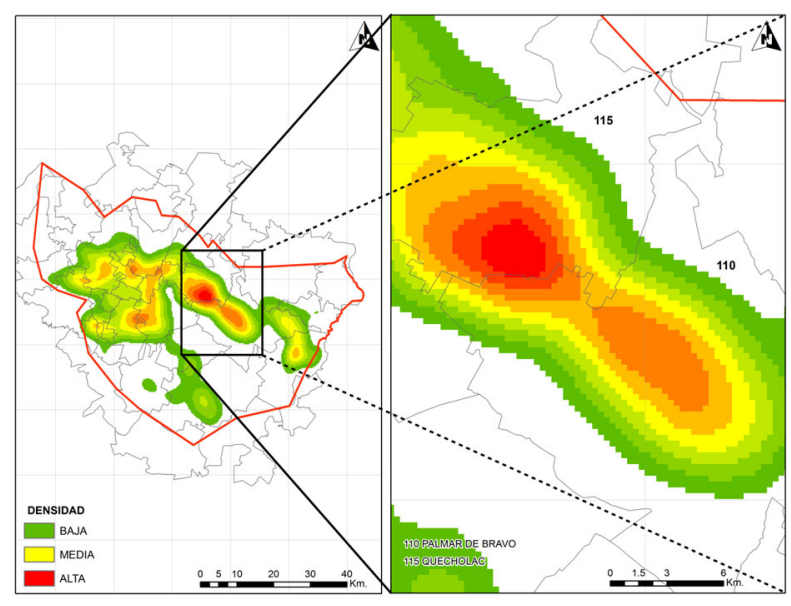

FIGURA 4

Zona de mayor densidad de aprovechamientos de agua subterránea de 1994 a 2014.

Fuente: elaboración propia

De los 1286 aprovechamientos de agua del AVT, el 78\% es destinado al uso agrícola, mismos que ocupan el $84 \%$ del volumen concesionado que corresponde $194223871 \mathrm{~m}^{3}$ anuales. El uso público urbano ocupa cerca del 16\%. El resto de los usos de agua asignados por el Repda (industrial, múltiple, domestico, pecuario y servicios) ocupan, en conjunto, solo el $2 \%$ respecto del total del volumen concesionado del acuífero (figuras 5 y 6$)$.

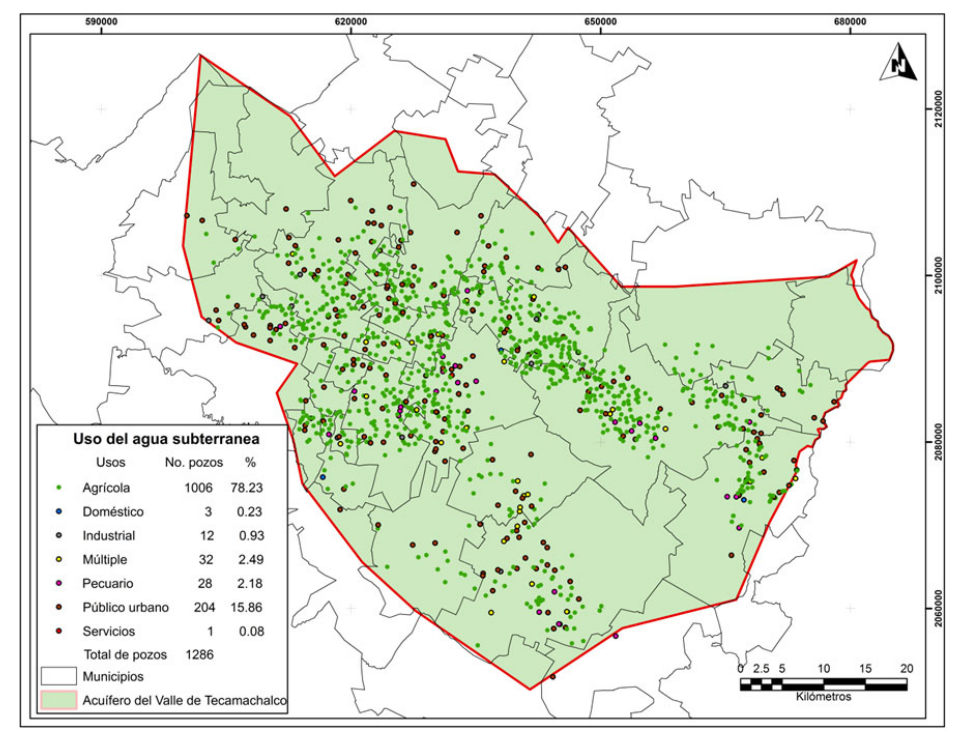

FIGURA 5.

Distribución geoespacial de los aprovechamientos de agua subterránea y sus usos. Fuente: elaboración propia con información del Repda de la Conagua. 


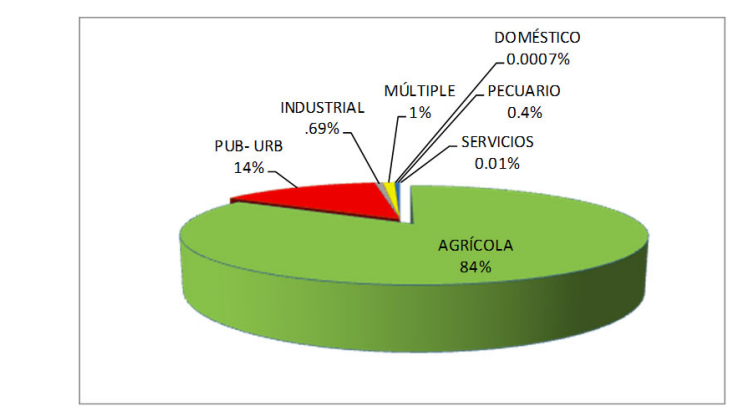

FIGURA 6.

Volumen concesionado por usos de agua del AVT.

Fuente: Elaboración propia.

\section{Discusión}

Los resultados que se presentan para el caso de este estudio están referidos al análisis de la tendencia e intensidad de extracción del recurso hídrico subterráneo en un espacio determinado, el AVT, que da pauta a una representación geográfica y esquemática que facilita su visualización y posterior análisis. Tal es el caso de la estimación de densidad Kernel, como lo refieren Moreno (1991) y Díaz, Bravo, Alatorre y Sánchez (2013, 2014).

La distribución y descripción de patrones espaciales a partir de la estimación de la densidad Kernel han sido utilizadas en diversos estudios, y aplicadas en diferentes áreas que han examinado problemas ecológicos (como la identificación de áreas de distribución de especies, y la contaminación de metales pesados y sistemas marinos); y problemáticas urbanas, como el acceso a servicios de salud, el crecimiento poblacional y la demanda de entornos urbanos, los accidentes de tránsito vehicular, la delincuencia y conflictos armados, entre otros (Gatrell et al., 1996; De Cos, 2004; Salas, 2007; Yu-Pin, Hone-Jay, Chen-Fa, Tsun-Kuo, \& Chiu-Yang, 2011; Kenchington et al., 2014). Este procedimiento es considerado como un método refinado de análisis de patrones espacial o de la distancia con el vecino más próximo (Rozas \& Camarero, 2005). Sin embargo, los estudios aplicados a los recursos hídricos son pocos. De forma particular, Díaz et al. (2013) aplicaron este método para identificar las regiones de mayor presión antropogénica sobre el agua subterránea en México desde una perspectiva geográfica.

La propiedad de un conjunto de datos situados en un área geográfica que muestran un patrón de organización o grado de agregación particular es útil para identificar concentraciones significativas de estos datos, de tal manera que validan la presencia de abundancia o disminución de ciertos atributos en áreas determinadas. Los resultados de la estimación de Kernel permiten un acercamiento a la situación actual del aprovechamiento del agua subterránea en el AVT.

Por su parte, el Repda registra volúmenes concesionados o asignados y los clasifica de acuerdo a sus usos consuntivos (extraen y consumen el agua desde su fuente de origen o abastecimiento), y no consuntivos (uso o reúso del agua en su ambiente natural sin necesidad de extracción o consumo). Díaz et al. (2013) señalan en su estudio que los datos del Repda pueden utilizarse como insumos de entrada para generar cartografía sobre la presión que se ejerce en el agua subterránea en México, y constituyen un referente para entender su condición actual y su evolución en el tiempo. Si bien la base de datos difiere geográficamente por los errores en la georreferenciación de los sitios o en la captura de datos de campo, permite una aproximación a la situación crítica que se tiene en cuanto a la sobreexplotación de los acuíferos del país, del estado de Puebla y, más específicamente, del AVT.

$\mathrm{Al}$ igual que en el resto de los países del mundo, en México la agricultura demanda una mayor cantidad de agua para el riego de cultivos. Barrionuevo y Feler (2014) señalan que el aumento de la superficie regada y, por 
ende, el mayor consumo de agua, podrían provocar en un futuro la profundización de los niveles freáticos, la disminución de la reserva de agua subterránea y el deterioro de la calidad de la misma (Shah, Molden, Sakthivadievel, \& Seckler, 2000). Esto evidencia la importancia de tener conocimiento de la evolución y la dinámica del uso y disponibilidad del agua. Evidentemente, el agua es uno de los recursos naturales más importantes para la agricultura. En el caso de México, se concesionaron los derechos del líquido a los usuarios, quienes se encargan de su administración y distribución.

Esta misma situación se presenta en el AVT, donde también se extrae el mayor volumen de agua para uso agrícola. Además de la agricultura, el sector público-urbano es el que demanda un mayor volumen concesionado. Al respecto, Díaz et al. (2014) señalan que la demanda social de agua para uso urbano se ha convertido en un factor crítico que limita el desarrollo de las regiones. La transferencia del líquido hacia las ciudades se torna prioritario, generalmente se transfiere agua de uso agrícola para uso urbano, lo que, en muchos casos, deteriora las áreas de cobertura de vegetación natural, las cuales, en gran medida, permiten la recarga de los acuíferos y, por tanto, coadyuvan a su agotamiento. Aunado a lo anterior, existen otros factores que contribuyen a la disminución de la recarga hídrica, como la deforestación y los cambios de uso de suelo, que afectan las zonas de recarga. Mientras tanto, los requerimientos de agua para diferentes propósitos se incrementan, por lo que todos los esfuerzos se deben centrar en el uso racional del agua disponible, tanto superficial como subterránea.

Es necesario complementar el estudio realizado con un análisis de las características específicas de las áreas más oscuras o de mayor densidad, conforme a los patrones definidos con la metodología de densidad Kernel. Además, llevar a cabo estudios hidrogeológicos regionales que indiquen con precisión el grado de afectación de los acuíferos, dado su nivel de extracción (Díaz et al., 2013). Aparte de considerar la baja eficiencia electromecánica de los equipos de bombeo, por su antigüedad y tiempo de operación, también se deben tener en cuenta obras de inversión para incrementar la eficiencia de los sistemas de riego y un adecuado manejo del agua subterránea de los acuíferos (Gutiérrez-Carrillo, Palacios-Vélez, Peña-Díaz, \& Palacios-Vélez, 2002).

Con la finalidad de incrementar la disponibilidad y productividad del agua, tanto en los sistemas como en las unidades de riego, se deben realizar, en el corto plazo, actividades de diagnóstico de funcionamiento de la infraestructura hidroagrícola y de evaluación de la eficiencia en el uso de los recursos, especialmente el agua, que mejorarán, en el mediano, plazo, la eficiencia en el uso de este recurso, desde la fuente de abastecimiento, el sistema de conducción y a nivel parcelario, es decir, a nivel de sistema o unidad (Villarreal-Manzo, 1994).

\section{Conclusiones}

La cartografía temática de distribución y uso de los aprovechamientos hídricos subterráneos — pozos profundos - en el área de influencia del AVT representan las variaciones en la distribución geográfica que definen las áreas de menor y mayor densidad de concentración de aprovechamientos y, por ende, de intensidad en la extracción de agua subterránea en el AVT en el estudio realizado, en el periodo comprendido de 1994 a 2014. La densidad Kernel permitió explicar la tendencia y el estado actual del aprovechamiento de agua por parte de los usuarios de los 27 municipios que conforman el acuífero y de las actividades productivas que se llevan a cabo en la zona, predominantemente la agrícola. El área de mayor densidad de aprovechamientos hídricos subterráneos se ubica en los municipios de Palmar de Bravo y Quecholac, donde el principal uso del agua está ligado a la agricultura. Así mismo, los patrones geoespaciales de los aprovechamientos evidencian la tendencia de consumo hacia un uso predominantemente público- urbano, principalmente en municipios con mayor número de habitantes como Tepeaca, Amozoc y Tecamachalco.

Si bien se requiere de estudios a profundidad que consideren el balance hídrico — superficial y subterráneo - del acuífero, en función de su capacidad de recarga natural y los volúmenes concesionados y, finalmente, extraídos, los resultados encontrados en el presente estudio son un referente para conocer y entender la 
condición actual del acuífero pero, sobre todo, para dimensionar su tendencia futura y tomar acciones tendentes a disminuir y frenar, en el mejor de los casos, tanto el abatimiento como la contaminación del AVT. Lo anterior implica la implementación de acciones en todos los niveles — de la sociedad beneficiaria, de las instituciones y empresas, de los técnicos y de los políticos y gobernantes - que consideren de forma urgente y prioritaria una planeación racional y un uso y manejo sustentable de los recursos hídricos superficiales y subterráneos disponibles, cada vez en menor cantidad, en el AVT.

\section{Notas}

Este artículo se deriva del proyecto de investigación Modelo Regional para el Manejo Sustentable del Agua del Acuifero del Valle de Tecamachalco, el cual fue desarrollado de enero de 2014 a julio de 2016, y corresponde a los autores Lucía Hernández Vivanco, Luis Alberto Villarreal Manzo, Benito Ramírez Valverde, Ignacio Ocampo Fletes, José Luis Jaramillo Villanueva y Benjamín Ortiz Espejel

\section{Referencias}

Barrionuevo, N. y Feler, M. (2014). Evolución del Área Regada por Pivote Central en la Provincia de Córdoba en el Período 1994-2014. Geografia y Sistemas de Información Geográfica (GEOSIG), 6(6), 133-144.

Carabias, J., Collado, J., Martínez, P., y Landa, R. (2005). Agua, medio ambiente y sociedad. Hacia la gestión integral de los recursos hidricos en México. México D.F.: Universidad Nacional Autónoma de México (UNAM), El Colegio de México, Fundación Gonzalo Rio Arronte.

Comisión Nacional del Agua (Conagua). (2003). Acuerdo por el que se dan a conocer los estudios técnicos del Acuífero 2101 Valle de Tecamachalco y se modifican los límites y planos de localización. Diario Oficial de la Federación.

Conagua. (2009). Acuerdo por el que se dan a Conocer los Estudios Técnicos del Acuífero 2101 Valle de Tecamachalco y se Modifican los Límites. Diario Oficial de la Federación. Recuperado de http://dof.gob.mx/nota_detalle_po pup.php?codigo $=5327360$.

Conagua. (2014a). Estadísticas del Agua en México. México D.F.: Secretaría del Medio Ambiente y Gobierno de la Republica.

Conagua. (2014b). Programa Nacional Hídrico 2014-2018. México D.F.: Secretaría del Medio Ambiente y Gobierno de la Republica.

De Cos, O. (2004). Valoración del método de densidades focales (Kernel) para identificación de patrones de crecimiento poblacional en España. Revista Internacional de Ciencia y Tecnología de la Información Geográfica (GeoFocus), 4, 136-165.

Díaz, R., Bravo, L., Alatorre, L., y Sánchez, E. (2013). Presión antropogénica sobre el agua subterránea en México: una aproximación geográfica. Investigaciones Geográficas, Boletin del Instituto de Geografía de la UNAM, 82, 93-103.

Díaz, R., Bravo, L., Alatorre, L. y Sánchez, E. (2014). Análisis geoespacial de la interacción entre el uso de suelo y de agua en el área periurbana de Cuauhtémoc, Chihuahua. Un estudio socioambiental en el norte de México. Investigaciones Geográficas, Boletin del Instituto de Geografia de la UNAM, 83, 116-130.

Gatrell, A., Bailey, T., Diggle, P., y Rowlingsont, S. (1996). Spatial point pattern analysis and its application in geographical epidemiology. The Royal Geographical Society, 21, 256-274.

Gibin, M., Longley, P., y Atkison, P. (2007). Kernel density estimation and percet volume contours in general practice catchment area analysis in urban areas. Geographical Information Science Research, UK Conference. National Centre for Geocomputation, National University of Ireland Maynooth and County Kildare, Ireland.

Gutiérrez-Carrillo, N., Palacios-Vélez, E., Peña-Díaz, S., y Palacios-Vélez, O. (2002). Escenarios para el aprovechamiento sustentable del acuífero del Valle de Querétaro. Agrociencia, 36, 1-10.

Guzmán-Soria, E., Hernández-Martínez, J., García-Salazar, J., Rebollar-Rebollar, S. De la Garza-Carranza, M., y Hernández-Soto, D. (2009). Consumo de agua subterránea en Guanajuato, México. Agrociencia, 43(7), 749-761. 
Instituto Nacional de Ecología (INE). (2005). Análisis del Subsidio a la Tarifa 09. Dirección General de Investigación en Política y Economía Ambiental.

Instituto Nacional de Estadística y Geografía (Inegi). (2010). Censo de Población y Vivienda 2010. Recuperado de ht tp://www.beta.inegi.org.mx/proyectos/ccpv/2010/

Kenchington, E., Murillo, F., Lirette, C., Sacau, M., Koen-Alonso, M., Kenny, A., ... Beazley, L. (2014). Kernel Density Surface Modelling as a Means to Identify Significant Concentrations of Vulnerable Marine Ecosystem Indicators. Plos One, 9(10). https://doi.org/10.1371/journal.pone.0109365

Llamas, M., Aldaya, M., Garrido, A., y López-Gunn, E. (2009). Soluciones para la escasez del agua en España y su aplicación a otras regiones. Revista de la Real Academia de Ciencias Exactas, Físicas y Naturales (Esp), 103(1), 41-54.

Moreno, A. (1991). Modelización cartográfica de densidades mediante estimadores Kernel. Treballs de la Societat Catalana de Geografia, 30, 445-460.

Rozas V. y Camarero, J. (2005). Técnicas de análisis espacial de patrones de puntos aplicadas en ecología forestal. Investigación Agraria: Sistemas y Recursos Forestales, 14(1), 79-97.

Salas, L. (2007). Identificación de patrones espaciales de la confrontación armada en Colombia con el método de densidades focales Kernel. 1998-2005. Perspectiva Geográfica, 12, 1-33

Secretaría de Medio Ambiente y Recursos Naturales (Semarnat). (2011). Estudios Técnicos de Aguas Nacionales Superficiales de la Región Hidrológica Número 18 Balsas. Diario Oficial de la Federación. Recuperado de http:/ /dof.gob.mx/nota_detalle.php?codigo $=5175730 \&$ fecha $=26 / 01 / 2011$

Semarnat. (2015). Acuerdo por el que se actualiza la disponibilidad media anual de agua subterránea de los 653 acuíferos de los Estados Unidos Mexicanos, mismos que forman parte de las regiones hidrológico-administrativas que se indican. Diario Oficial de La Federación. Recuperado de https://dof.gob.mx/nota_detalle.php?codigo=551004 $2 \&$ fecha $=04 / 01 / 2018$

Shah, T., Molden, D., Sakthivadievel, R., y Seckler, D. (2000). Global groundwater situation: opportunities and challenges. Economic and Political Weekly, 36(43), 4142-4150.

Villarreal-Manzo, L. (1994). Metodologías de Diagnóstico y planeación de la operación de unidades de riego por bombeo (Tesis de Maestría). Colegio de Postgraduados, Texcoco, México.

Yu-Pin, L., Hone-Jay C., Chen-Fa W., Tsun-Kuo C., y Chiu-Yang C. (2011). Hotspot analysis of spatial environmental pollutants using Kernel density estimation and geostatistical techniques. International Journal of Environmental Research and Public Health, 8, 75-88.

\section{Licencia Creative Commons CC BY 4.0}

Cómo citar este artículo: Hernández, L., Villarreal, L. A., Ramírez, B., Ocampo, I., Jaramillo, J. L., y Ortiz, B. (2018). Distribución espacial y temporal de aprovechamientos de agua del acuífero del Valle de Tecamachalco, Puebla. Ambiente Y Desarrollo, 22(42), 1-11. https://doi.org/10.11144/Javeriana.ayd22-42.deta 